\title{
A NOTE ON SOME TOPOLOGICAL PROPERTIES OF SETS WITH FINITE PERIMETER
}

\author{
SILVANO DELLADIO
}

\begin{abstract}
Some well-known results about the 2-density topology on $\mathbb{R}$ (in particular in the context of the Lusin-Menchoff property) are extended to $\tau_{b_{m}}$, i.e. the $m$-density topology on $\mathbb{R}^{n}$ with $m \in(n,+\infty)$. Every set of finite perimeter in $\mathbb{R}^{n}$ is equivalent (in measure) to a set in $\tau_{b_{m_{0}}}$, where $m_{0}=n+1+\frac{1}{n-1}$. There exists a set of finite perimeter in $\mathbb{R}^{n}$ which is not equivalent (in measure) to any member in the a.e.-modification of $\tau_{b_{m}}$, whatever $m \in[n,+\infty)$.
\end{abstract}

\section{INTRODUCTION}

It is increasingly clear that, in many regards, the sets of finite perimeter behave as open sets and several recent achievement support this idea. In particular it is worth mentioning the following results: the decomposition property and the connectedness by arcs of indecomposable plane sets [2, Theorem 1 and Lemma 6, Theorem 8], the constancy property [11, Lemma A.2], the superdensity property [5, Lemma 4.1] and the infinitesimal euclideanity [8, Theorem 3.2]. The closure property for integral currents [12, Theorem 1], in the special case when the current is associated to a set of finite perimeter, must be included in the list too. In fact, we expect that many of the results above can be extended in some way to integral currents. Moreover, many applications of these properties have been provided, e.g. in $[2,4,5,9,10,11,13,14,16]$.

This work must be regarded as a contribution to the study of the geometry of sets of finite perimeter. Its main objective is to begin the exploration of the connection between the theory of sets of finite perimeter and fine topology methods, whose existence came to light with the discovery of the mentioned superdensity property [5, Lemma 4.1]. Fine topology methods have several applications in the theory of real functions and in potential theory. With regards to these applications, one of the most useful tools is a binormality condition known as the "Lusin-Menchoff property", compare Definition 3.2. It plays a key role in many results about various type of finely continuous functions (e.g. approximately continuous functions) and purely topological properties of harmonic spaces (e.g. the theory of hyperharmonic functions and of fine Dirichlet problem).

2010 Mathematics Subject Classification. Primary 28A75, 54-XX; .

Key words and phrases. Sets of finite perimeter, Superdensity, Fine topology methods, Lusin-Menchoff property. 
In this paper we are especially interested into the operator $b_{m}: \mathcal{P}\left(\mathbb{R}^{n}\right) \rightarrow \mathcal{P}\left(\mathbb{R}^{n}\right)$, where $m$ is a real number greater or equal to $n$, which is defined as follows:

$$
b_{m}(A):=\left\{x \in \mathbb{R}^{n} \mid \limsup _{r \rightarrow 0+} \frac{\mathcal{L}^{n}(A \cap B(x, r))}{r^{m}}>0\right\}, \quad A \in \mathcal{P}\left(\mathbb{R}^{n}\right) .
$$

We note that $\left[b_{m}\left(A^{c}\right)\right]^{c}$ is the set of points $x$ in $\mathbb{R}^{n}$ such that $\mathcal{L}^{n}(B(x, r) \backslash A)=o\left(r^{m}\right)$, as $r \rightarrow 0+$. This simple observation leads to the idea of defining a "superdensity topology": just as we say that $A$ is open in the Euclidean topology whenever $A \subset A^{\circ}$, so we'll say that $A$ is open with respect to $b_{m}$ if $A \subset\left[b_{m}\left(A^{c}\right)\right]^{c}$. This idea concerns a particular case of a very general and deep theory about fine topologies arising from a base operator. The most complete reference for this subject is [15], from which we recall that a base operator on a set $X$ is a map $b: \mathcal{P}(X) \rightarrow \mathcal{P}(X)$ such that $b(\emptyset)=\emptyset$ and $b(A \cup B)=b(A)+b(B)$, for all $A, B \in \mathcal{P}(X)$. Such an operator is monotone and it actually determines a topology $\tau_{b}$ whose members are the subsets $A$ of $X$ satisfying $A \subset\left[b\left(A^{c}\right)\right]^{c}$. Hence a subset $F$ of $X$ is closed with respect $\tau_{b}$ (or $b$-closed, for shortness) if and only if $b(F) \subset F$.

The book [15] does not deal explicitly with $b_{m}$, for all $n$ and $m$ (with $m \in[n,+\infty)$ ), but presents a very detailed study of $b_{2}$ in $\mathbb{R}$, as well as a partial investigation of a certain base operator on a metric space which generalizes $b_{m}$ (compare $[15,6.28]$ ). Recovering the properties of $b_{m}$ and $\tau_{b_{m}}$ from the theory in [15] is a long and not always easy exercise. For this reason, the series of results discussed in Section 3 below is to some extent a "guided tour" within [15]. As for the proofs, we only provide those that are not related in a simple way to the ones stated in the book.

As we shall prove in Theorem 3.1, if $m \in(n,+\infty)$ then $\tau_{b_{m}}$ has not the Lusin-Menchoff property. The proof replicates the argument used in $[15,6.27(\mathrm{j})]$ for the special case $n=1$ and $m=2$, except for some non completely trivial steps. In particular, we need Proposition 3.2 which states roughly this: If $m \in(n,+\infty)$ and $A$ is sufficiently diffused within an open set, then $b_{m}(A)$ may include this open set, regardless of how the measure of $A$ is small. About Proposition 3.2, it is worth noting that:

- For $m \geq n+1$, it can be proved by combining the Alberti's Lusin-type theorem [1, Theorem 1] and the closure result [5, Theorem 2.1], compare [6, Theorem 1.5]. We observe that this is an argument within the framework of potential theory. As another example of application of [5, Theorem 2.1] in the same context, we mention the simplified proof of [12, Corollary 2] given in [6];

- Surprisingly, the maximum order of density common to all sets of finite perimeter, namely $m_{0}:=n+1^{*}$ (where $1^{*}$ is the Sobolev conjugate of 1 ), "emerges spontaneously" in the course of the proof of the first assertion. More precisely, it turns out that $A$ has finite perimeter as soon as $m$ exceeds such a number.

A topology which has the Lusin-Menchoff property and is strictly related to the superdensity topology is the so called "a.e.-modification" of $\tau_{b_{m}}$. It is determined by the base operator defined as $a_{m}(A):=b_{m}(\bar{A}), A \in \mathcal{P}\left(\mathbb{R}^{n}\right)$, hence one has $\tau_{a_{m}} \subset \tau_{b_{m}}$. As we prove 
in Proposition 4.2, every set $E$ of finite perimeter is inner-regular with respect to $\tau_{b_{m_{0}}}$, namely there exists $G \in \tau_{b_{m_{0}}}$ such that $G \subset E$ and $\mathcal{L}^{n}(G)=\mathcal{L}^{n}(E)$. Based on this fact, one might expect that a similar regularity property may also exist with respect to the topology $\tau_{a_{m_{0}}}$. Unfortunately this does not happen and in fact Proposition 4.3 provides an example of set with finite perimeter which is not equivalent (in measure) to any member of $\tau_{a_{m}}$, whatever $m \in[n,+\infty)$.

\section{General notation}

The Euclidean norms (absolute value included) are denoted by $|\cdot|$. The open ball of radius $r$ centered at $x \in \mathbb{R}^{n}$ is denoted by $B(x, r)$ and $\omega_{n}$ indicates the measure of the unit ball $B(0,1)$. For simplicity, the ball $B(0, r)$ is indicated with $B_{r}$. The interior of a set $E$ is denoted by $E^{\circ}$. The constants depending only on $p, q, \ldots$ are indicated by $C(p, q, \ldots)$. The usual Euclidean topology in $\mathbb{R}^{n}$ is indicated with $\tau\left(\mathbb{R}^{n}\right)$. If $E \subset \mathbb{R}^{n}$ then $\bar{E}$ denotes the closure of $E$ with respect to $\tau\left(\mathbb{R}^{n}\right)$. $\mathcal{L}^{n}$ is the Lebesgue outer measure on $\mathbb{R}^{n}$. With $P(E)$ we denote the perimeter of a subset $E$ of $\mathbb{R}^{n}$, in the sense of De Giorgi [3, Section 3.3].

\section{Some PROPERTIES OF THE SUPERDENSITY TOPOLOGY ON $\mathbb{R}^{n}$}

Let us begin this section by recalling the definition of $m$-density point which has been defined and studied in the papers $[5,6,7]$.

Definition 3.1. Let $A \in \mathcal{P}\left(\mathbb{R}^{n}\right)$ and $m \in[n,+\infty)$. Then $x \in \mathbb{R}^{n}$ is said to be a " $m$ density point of $A$ " if

$$
\lim _{r \rightarrow 0+} \frac{\mathcal{L}^{n}(B(x, r) \backslash A)}{r^{m}}=0 .
$$

The set of $m$-density points of $A$ is denoted by $A^{(m)}$.

Remark 3.1. As we observed in the introduction, one has the equality

$$
A^{(m)}=\left[b_{m}\left(A^{c}\right)\right]^{c} .
$$

Note that the map $A \mapsto A^{(m)}, A \in \mathcal{P}\left(\mathbb{R}^{n}\right)$, is not a base operator on $\mathbb{R}^{n}$. Indeed, let $A$ be an open set with Lipschitz boundary and let $B:=\mathbb{R}^{n} \backslash \bar{A}$. Then one obviously has $A^{(m)}=A, B^{(m)}=B$ and $(A \cup B)^{(m)}=\mathbb{R}^{n}$, hence $A^{(m)} \cup B^{(m)}$ is a proper subset of $(A \cup B)^{(m)}$.

Proposition 3.1. The following facts hold:

(1) $b_{m}$ is a base operator;

(2) For all $A \subset \mathbb{R}^{n}$, the set $b_{m}(A)$ is measurable with respect to $\mathcal{L}^{n}$. As a consequence, $A^{(m)}$ is measurable too;

(3) One has $A \in \tau_{b_{m}}$ if and only if $A \subset A^{(m)}$. In particular $\tau_{b_{m}}$ is finer than $\tau\left(\mathbb{R}^{n}\right)$; 
(4) If $p \geq m(\geq n)$ then $b_{m}(A) \subset b_{p}(A)$, for all $A \subset \mathbb{R}^{n}$. In particular $\tau_{b_{m}}$ is finer than $\tau_{b_{p}}$

(5) For all $A \subset \mathbb{R}^{n}$, one has

$$
b_{m}(A) \subset \bar{A}^{\tau_{b_{m}}}
$$

where the right hand member is the closure of $A$ with respect to $\tau_{b_{m}}$. In particular, a set $A$ is $\tau_{b_{m}}$-dense in $\mathbb{R}^{n}$ whenever $b_{m}(A)=\mathbb{R}^{n}$.

Proof. (1) Compare [15, 6.28].

(2) For all $x, y \in \mathbb{R}^{n}, r>0$ and $A \in \mathcal{P}\left(\mathbb{R}^{n}\right)$, one has

$$
\mathcal{L}^{n}(B(x, r) \cap A)=\mathcal{L}^{n}(B(x, r) \cap B(y, r) \cap A)+\mathcal{L}^{n}([B(x, r) \backslash B(y, r)] \cap A)
$$

and

$$
\mathcal{L}^{n}(B(y, r) \cap A)=\mathcal{L}^{n}(B(y, r) \cap B(x, r) \cap A)+\mathcal{L}^{n}([B(y, r) \backslash B(x, r)] \cap A)
$$

hence

$$
\left|\mathcal{L}^{n}(B(x, r) \cap A)-\mathcal{L}^{n}(B(y, r) \cap A)\right| \leq \mathcal{L}^{n}(B(x, r) \triangle B(y, r)) .
$$

It follows that $x \mapsto \mathcal{L}^{n}(B(x, r) \cap A)$ is continuous (in fact it is Lipschitz!), so that the function

$$
x \mapsto \limsup _{r \rightarrow 0+} \frac{\mathcal{L}^{n}(A \cap B(x, r))}{r^{m}}
$$

has to be measurable. But $b_{m}(A)$ is the inverse image of $(0,+\infty]$ under this function, thus it has to be measurable.

(3) Recall that $A \in \tau_{b_{m}}$ if and only if $b_{m}\left(A^{c}\right) \subset A^{c}$. Then the assertion follows at once from (3.1).

(4) It's obvious, by (1.1).

(5) If $A \subset \mathbb{R}^{n}$ and $F$ is any $b_{m}$-closed set (i.e. one has $b_{m}(F) \subset F$ ) including $A$, then

$$
b_{m}(A) \subset b_{m}(F) \subset F .
$$

The conclusion follows from the arbitrariness of $F$.

The following result generalizes the properties (d) and (e) pointed out in [15, 6.27].

Proposition 3.2. Let $m \in(n,+\infty)$ and consider $\varepsilon>0$. The following properties hold:

(1) If $\Omega$ is a bounded open set in $\mathbb{R}^{n}$, then there exists an open subset $A$ of $\Omega$ such that

$$
\mathcal{L}^{n}(A)<\varepsilon, \quad \Omega \subset b_{m}(A) \subset \bar{\Omega} .
$$

In the special case when $\partial \Omega$ is Lipschitz, the set $A$ can be chosen in such a way to satisfy

$$
b_{m}(A)=\bar{\Omega}
$$


(2) There exists an open subset $U$ of $\mathbb{R}^{n}$ such that

$$
\mathcal{L}^{n}(U)<\varepsilon, \quad b_{m}(U)=\mathbb{R}^{n} .
$$

Proof. (1) Let $R$ and $\beta$ be positive numbers such that

$$
\bar{\Omega} \subset B_{R}, \quad 2^{n} R^{n} \geq 1, \quad\left(2^{n} R^{n}+1\right)^{\frac{1}{m-n}} \geq 2
$$

and

$$
\beta>\max \left\{\left(2^{n} R^{n}+1\right)^{\frac{1}{m-n}},\left(\frac{\varepsilon}{\omega_{n}}\right)^{1 / n}+\frac{n^{1 / 2}}{2}\right\} .
$$

Also define (for $h=1,2, \ldots$ )

$$
\rho_{h}:=\left(\frac{\varepsilon}{\omega_{n}}\right)^{\frac{1}{n}} \beta^{-\frac{h m}{n}}
$$

and let $\Lambda_{h}$ denote the lattice of step $\beta^{-h}$ (in $\mathbb{R}^{n}$ ), i.e. $\Lambda_{h}:=\beta^{-h} \mathbb{Z}^{n}$. Then put

$$
\Gamma_{h}:=\left\{P \in \Lambda_{h} \mid B\left(P, \rho_{h}\right) \subset \Omega\right\}, \quad A_{h}:=\bigcup_{P \in \Gamma_{h}} B\left(P, \rho_{h}\right), \quad A^{\prime}:=\bigcup_{h=1}^{+\infty} A_{h} .
$$

and observe that

$$
\#\left(\Gamma_{h}\right) \leq\left(\frac{2 R}{\beta^{-h}}\right)^{n}=2^{n} R^{n} \beta^{n h} .
$$

Hence, recalling also (3.4), we get

$$
\begin{aligned}
\mathcal{L}^{n}\left(A^{\prime}\right) & \leq \sum_{h=1}^{+\infty} \mathcal{L}^{n}\left(A_{h}\right) \leq \sum_{h=1}^{+\infty} \#\left(\Gamma_{h}\right) \omega_{n} \rho_{h}^{n} \\
& \leq 2^{n} R^{n} \varepsilon \sum_{h=1}^{+\infty} \beta^{(n-m) h}=\frac{2^{n} R^{n} \varepsilon}{\beta^{m-n}-1} \\
& <\varepsilon .
\end{aligned}
$$

Let us prove that

$$
\Omega \subset b_{m}\left(A^{\prime}\right) .
$$

To this aim, consider $x \in \Omega$. Then choose $H_{x}>0$ such that

$$
B\left(x, \beta^{-H_{x}}\right) \subset \Omega
$$

and observe that for every $h \geq H_{x}$ there must be $P_{h+1} \in \Lambda_{h+1}$ satisfying

$$
\left|x-P_{h+1}\right| \leq \frac{\beta^{-(h+1)} n^{1 / 2}}{2} .
$$

Then, for all $h \geq H_{x}$ and $y \in B\left(P_{h+1}, \rho_{h+1}\right)$, we find

$$
|y-x| \leq\left|y-P_{h+1}\right|+\left|P_{h+1}-x\right|<\rho_{h+1}+\frac{\beta^{-(h+1)} n^{1 / 2}}{2}<\left[\left(\frac{\varepsilon}{\omega_{n}}\right)^{1 / n}+\frac{n^{1 / 2}}{2}\right] \beta^{-(h+1)}
$$

hence, by recalling once again (3.4)

$$
|y-x|<\beta^{-h}
$$


This proves that for all $h \geq H_{x}$ one has

$$
B\left(P_{h+1}, \rho_{h+1}\right) \subset B\left(x, \beta^{-h}\right) .
$$

From (3.8) and (3.9) it follows that $P_{h+1} \in \Gamma_{h+1}$ and then also

$$
B\left(P_{h+1}, \rho_{h+1}\right) \subset A_{h+1} \subset A^{\prime} .
$$

Now, by (3.9) and (3.10), we obtain

$$
\frac{\mathcal{L}^{n}\left(A^{\prime} \cap B\left(x, \beta^{-h}\right)\right)}{\left(\beta^{-h}\right)^{m}} \geq \frac{\mathcal{L}^{n}\left(B\left(P_{h+1}, \rho_{h+1}\right)\right)}{\beta^{-m h}}=\omega_{n} \rho_{h+1}^{n} \beta^{m h}=\frac{\varepsilon}{\beta^{m}}
$$

for all $h \geq H_{x}$, hence (3.7).

We complete the proof of (3.2) by observing that $A^{\prime} \subset \Omega \subset \bar{\Omega}$ and that $\bar{\Omega}$ is closed with respect to the $b_{m}$-topology (by (3) of Proposition 3.1), whereby

$$
b_{m}\left(A^{\prime}\right) \subset b_{m}(\bar{\Omega}) \subset \bar{\Omega} .
$$

Finally assume that $\partial \Omega$ is Lipschitz. Then consider an open set $A^{\prime \prime}$ satisfying

$$
A^{\prime \prime} \supset B_{R} \backslash \Omega, \quad \mathcal{L}^{n}\left(A^{\prime \prime} \backslash\left[B_{R} \backslash \Omega\right]\right)<\varepsilon-\mathcal{L}^{n}\left(A^{\prime}\right) .
$$

Observe that

$$
A^{\prime \prime} \cap \Omega \subset A^{\prime \prime} \backslash\left[B_{R} \backslash \Omega\right]
$$

and define

$$
A:=A^{\prime} \cup\left(A^{\prime \prime} \cap \Omega\right) \quad(\text { which is a subset of } \Omega) \text {. }
$$

Then

$$
\mathcal{L}^{n}(A) \leq \mathcal{L}^{n}\left(A^{\prime}\right)+\mathcal{L}^{n}\left(A^{\prime \prime} \cap \Omega\right) \leq \mathcal{L}^{n}\left(A^{\prime}\right)+\mathcal{L}^{n}\left(A^{\prime \prime} \backslash\left[B_{R} \backslash \Omega\right]\right)<\varepsilon .
$$

It remains to prove that $b_{m}(A)=\bar{\Omega}$. As for the inclusion

$$
b_{m}(A) \subset \bar{\Omega}
$$

observe that it follows at once from the same argument used to prove (3.11). On the other hand

$$
b_{m}(A) \supset b_{m}\left(A^{\prime}\right) \supset \Omega
$$

follows by the monotonicity of $b_{m}$ (recalling that $A^{\prime} \subset A$ ) and (3.7). So we are reduced to prove that

$$
\partial \Omega \subset b_{m}(A) .
$$

To this aim, consider $x \in \partial \Omega$. Since $\partial \Omega \subset A^{\prime \prime}$, for $r$ small enough one has $B(x, r) \subset A^{\prime \prime}$ hence

$$
A \cap B(x, r)=\left[A^{\prime} \cup\left(A^{\prime \prime} \cap \Omega\right)\right] \cap B(x, r) \supset A^{\prime \prime} \cap \Omega \cap B(x, r)=\Omega \cap B(x, r) .
$$

But $\partial \Omega$ is Lipschitz, thus there exists $C>0$ such that

$$
\mathcal{L}^{n}(A \cap B(x, r)) \geq C r^{n} \geq C r^{m}
$$

provided $r$ is small enough, which proves (3.13). 
(2) Let $\left\{\Omega_{j}\right\}$ be a countable family of bounded open subsets of $\mathbb{R}^{n}$ such that

$$
\bigcup_{j} \Omega_{j}=\mathbb{R}^{n}
$$

Then, by (1), for every $j=1,2, \ldots$ there exists an open set $A_{j}$ such that

$$
A_{j} \subset \Omega_{j}, \quad \mathcal{L}^{n}\left(A_{j}\right)<\frac{\varepsilon}{2^{j}}, \quad \Omega_{j} \subset b_{m}\left(A_{j}\right) .
$$

Define

$$
U:=\bigcup_{j} A_{j}
$$

Then $U$ is open and $\mathcal{L}^{n}(U)<\varepsilon$. Finally, by the monotonicity of $b_{m}$, one has

$$
b_{m}(U) \supset b_{m}\left(\cup_{j=1}^{N} A_{j}\right)=\bigcup_{j=1}^{N} b_{m}\left(A_{j}\right) \supset \bigcup_{j=1}^{N} \Omega_{j}
$$

for all $N \geq 1$, hence $b_{m}(U)=\mathbb{R}^{n}$.

Remark 3.2. One can easily guess that the set $A^{\prime}$ considered in the proof of Proposition 3.2 must have finite perimeter, provided that $m$ is large enough. More precisely, we can prove that this fact occurs for $m>m_{0}$, with

$$
m_{0}:=\frac{n^{2}}{n-1}=n+1+\frac{1}{n-1} .
$$

Indeed, by (3.5), one has

$$
A_{H}^{\prime}:=\bigcup_{h=1}^{H} A_{h} \rightarrow A^{\prime}
$$

in $L^{1}$, as $H \rightarrow \infty$. Hence, by [3, Proposition 3.38] and (3.6), we get

$$
P\left(A^{\prime}\right) \leq \liminf _{H \rightarrow \infty} P\left(A_{H}^{\prime}\right) \leq \sum_{h=1}^{+\infty} \#\left(\Gamma_{h}\right) n \omega_{n} \rho_{h}^{n-1} \leq C(n, R, \varepsilon) \sum_{h=1}^{+\infty} \beta^{n h-\frac{h m(n-1)}{n}}
$$

namely

$$
P\left(A^{\prime}\right) \leq C(n, R, \varepsilon) \sum_{h=1}^{+\infty}\left[\beta^{\frac{n-1}{n}\left(m_{0}-m\right)}\right]^{h}<+\infty
$$

which proves our assertion. Observe that $m_{0}=n+1^{*}$ (where $1^{*}$ is the Sobolev conjugate of 1) and that $m_{0}$ is the maximum order of density which is common to all sets of finite perimeter, according to Theorem 4.1 and Proposition 4.1 below.

Remark 3.3. Under the stronger assumption $m \geq n+1$, the statement (2) of Proposition 3.2 has been proved in [6, Theorem 1.5] by an easy argument combining the Alberti's Lusin-type theorem [1, Theorem 1] and the closure result [5, Theorem 2.1].

Now let us recall the Lusin-Menchoff property for a fine topology on $\mathbb{R}^{n}$, compare [15]. 
Definition 3.2. Let $\tau^{\prime}$ be a topology on $\mathbb{R}^{n}$ such that $\tau^{\prime}$ is finer than $\tau\left(\mathbb{R}^{n}\right)$. We say that " $\tau^{\prime}$ has the Lusin-Menchoff property" if for all $\tau\left(\mathbb{R}^{n}\right)$-closed set $F$ and $\tau^{\prime}$-closed set $F^{\prime}$ satisfying $F \cap F^{\prime}=\emptyset$ there exist $G \in \tau\left(\mathbb{R}^{n}\right)$ and $G^{\prime} \in \tau^{\prime}$ such that

$$
G \supset F^{\prime}, \quad G^{\prime} \supset F, \quad G \cap G^{\prime}=\emptyset .
$$

Remark 3.4. The argument given in [15] to prove that the topology $\tau_{b_{2}}$ on $\mathbb{R}$ has not the Lusin-Menchoff property can be easily extended to the topology $\tau_{b_{m}}$ on $\mathbb{R}^{n}$ with $m>n$. More precisely, in two steps:

- One replicates the argument in (j) of [15, 6.27], using Proposition 3.2 instead of (e) of $[15,6.27]$ and recalling (5) of Proposition 3.1. One gets:

Proposition 3.3. If $m \in(n,+\infty)$ then $\left(\mathbb{R}^{n}, \tau_{b_{m}}\right)$ is not a Baire space, namely there is a nonempty open set (with respect to $\tau_{b_{m}}$ ) which is the union of countably many nowhere dense sets (with respect to $\tau_{b_{m}}$ ).

- From Proposition 3.3 and [15, Proposition 3.16] one finally obtains:

Theorem 3.1. If $m \in(n,+\infty)$ then $\tau_{b_{m}}$ has not the Lusin-Menchoff property.

A fine topology on $\mathbb{R}^{n}$ which is strictly related to $\tau_{b_{m}}$ and turns out to have the LusinMenchoff property is the one determined by the base operator known as "the $a$-modification of $b_{m}$ " (compare [15, Section 7.1]). Such a fact is established as the second statement of the following result and is a trivial corollary of [15, Theorem 6.33] (with $m^{*}=\mathcal{L}^{n}, b=b_{m}$, $\omega(r)=r^{m+1}$ ) and [15, Theorem 7.5]. The first statement, instead, follows at once from [15, Theorem 7.4].

Proposition 3.4. For $m \in[n,+\infty)$ let $a_{m}: \mathcal{P}\left(\mathbb{R}^{n}\right) \rightarrow \mathcal{P}\left(\mathbb{R}^{n}\right)$ be the a-modification of $b_{m}$, i.e. the base operator defined by

$$
a_{m}(A):=b_{m}(\bar{A}), \quad A \in \mathcal{P}\left(\mathbb{R}^{n}\right) .
$$

Then:

(1) $\tau_{a_{m}}$ coincides with the a.e.-modification of $\tau_{b_{m}}$, i.e. it is generated by the family of all sets in $\tau_{b_{m}}$ of the form $U \cup\{x\}$, with $U \in \tau\left(\mathbb{R}^{n}\right)$ and $x \in \mathbb{R}^{n}$, together with the empty set;

(2) $\tau_{a_{m}}$ has the Lusin-Menchoff property.

Remark 3.5. We observe that the application of [15, Theorem 6.33] (with $m^{*}=\mathcal{L}^{n}$, $b=b_{m}, \omega(r)=r^{m+1}$ ) leads to prove that the superdensity base operator $b_{m}$ has the complete Lusin-Menchoff property in the sense of [15, Section 3.C].

The next proposition provides a characterization of the $\tau_{a_{m}}$-open sets.

Proposition 3.5. Let $m \in[n,+\infty)$. Then $A \in \tau_{a_{m}}$ if and only if $A \subset\left(A^{\circ}\right)^{(m)}$. 
Proof. First of all prove that, for all $A \in \mathcal{P}\left(\mathbb{R}^{n}\right)$, one has

$$
\overline{A^{c}}=\left(A^{\circ}\right)^{c} .
$$

Indeed:

- From $A^{\circ} \subset A$ we get $A^{c} \subset\left(A^{\circ}\right)^{c}$, hence $\overline{A^{c}} \subset \overline{\left(A^{\circ}\right)^{c}}=\left(A^{\circ}\right)^{c}$;

- As for the opposite inclusion, observe that

$$
A \backslash A^{\circ} \subset \bar{A} \backslash A^{\circ}=\partial A=\bar{A} \cap \overline{A^{c}} \subset \overline{A^{c}}
$$

hence the conclusion follows by the equality $\left(A^{\circ}\right)^{c}=\left(A \backslash A^{\circ}\right) \cup\left(A^{c} \backslash A^{\circ}\right)$.

Now, by (3.14) and (3.1), we obtain

$$
a_{m}\left(A^{c}\right)=b_{m}\left(\overline{A^{c}}\right)=b_{m}\left(\left(A^{\circ}\right)^{c}\right)=\left[\left(A^{\circ}\right)^{(m)}\right]^{c}
$$

for all $A \in \mathcal{P}\left(\mathbb{R}^{n}\right)$. The conclusion follows readily by recalling that $A \in \tau_{a_{m}}$ if and only if $a_{m}\left(A^{c}\right) \subset\left(A^{c}\right)$.

Remark 3.6. Proposition 3.5 and (3) of Proposition 3.1 yield

$$
\tau\left(\mathbb{R}^{n}\right) \subset \tau_{a_{m}} \subset \tau_{b_{m}}
$$

and both inclusions are proper, as the following examples show:

- Let $A:=\mathbb{R}^{n} \backslash H$, where $H$ is an open half-line. Then $\left(A^{\circ}\right)^{(m)}=\mathbb{R}^{n}$, hence $A \in \tau_{a_{m}} \backslash \tau\left(\mathbb{R}^{n}\right)$;

- Let $A:=\mathbb{R}^{n} \backslash \mathbb{Q}^{n}$. Since $\left(A^{\circ}\right)^{(m)}=\emptyset$ and $A^{(m)}=\mathbb{R}^{n}$, one has $A \in \tau_{b_{m}} \backslash \tau_{a_{m}}$.

\section{A FAmily of Superdense SETS: Sets of Finite PERIMeter}

A celebrated theorem by Lebesgue states that if $E$ is a measurable subset of $\mathbb{R}^{n}$ then almost every $x \in E$ is a $n$-density point of $E$. The following result, proved in [5], establishes that Caccioppoli sets are more dense than generic measurable sets.

Theorem 4.1. Let $E$ be a subset of $\mathbb{R}^{n}$ with finite perimeter and

$$
m_{0}:=n+1+\frac{1}{n-1} \text {. }
$$

Then $\mathcal{L}^{n}\left(E \backslash E^{\left(m_{0}\right)}\right)=0$.

The following proposition shows that $m_{0}$ is the maximum order of density which is common to all sets of finite perimeter.

Proposition 4.1. Let $m \in\left(m_{0},+\infty\right)$, with $m_{0}$ defined by (4.1). Then there exists a closed set $F_{m}$ of positive measure and finite perimeter in $\mathbb{R}^{n}$ such that $F_{m}^{(m)}=\emptyset$. 
Proof. Let us go along the lines of the proof of Proposition 3.2, by starting with $\Omega:=B_{1}$ and choosing $A^{\prime \prime}$ to be an annulus centered at 0. From (3.12) and [3, Proposition 3.38] it follows that

$$
P(A) \leq P\left(A^{\prime}\right)+P\left(A^{\prime \prime}\right)+P(\Omega)
$$

whereby, since Remark 3.2, the open set $A$ has finite perimeter. Hence the closed set

$$
F_{m}:=A^{c} \cap \bar{\Omega}
$$

has finite perimeter too. Recalling Definition 1.1 and (3.3), we finally get

$$
\left(F_{m}^{(m)}\right)^{c}=b_{m}\left(\left(F_{m}\right)^{c}\right)=b_{m}\left(A \cup(\bar{\Omega})^{c}\right)=b_{m}(A) \cup b_{m}\left((\bar{\Omega})^{c}\right)=\bar{\Omega} \cup \Omega^{c}=\mathbb{R}^{n} .
$$

Let us prove that every set of finite perimeter has an equivalent copy (with respect to measure) in $\tau_{b_{m_{0}}}$.

Proposition 4.2. Let $E$ be a set of finite perimeter in $\mathbb{R}^{n}$ and define

$$
E_{*}:=E \cap E^{\left(m_{0}\right)} \text {. }
$$

with $m_{0}$ defined by (4.1). Then

$$
\mathcal{L}^{n}\left(E_{*}\right)=\mathcal{L}^{n}(E), \quad E_{*} \in \tau_{b_{m_{0}}} .
$$

Proof. The first equality follows at once from Theorem 4.1 and (2) of Proposition 3.1:

$$
\mathcal{L}^{n}(E)=\mathcal{L}^{n}\left(E \cap E^{\left(m_{0}\right)}\right)+\mathcal{L}^{n}\left(E \backslash E^{\left(m_{0}\right)}\right)=\mathcal{L}^{n}\left(E_{*}\right) .
$$

Hence one also has

$$
E_{*}^{\left(m_{0}\right)}=E^{\left(m_{0}\right)} \supset E_{*}
$$

namely $E_{*} \in \tau_{b_{m_{0}}}$, by (3) of Proposition 3.1 .

In view of Proposition 3.4, it would be interesting to know that every set of finite perimeter has a copy in $\tau_{a_{m}}$ for some $m>n$. Unfortunately, this fact doesn't take place as evidenced by the following result.

Proposition 4.3. There exists a subset $E$ of $\mathbb{R}^{n}$ with the following properties:

(1) $E$ is closed and $E^{\circ}=\emptyset$;

(2) E has positive measure and finite perimeter;

(3) One cannot find $m \in[n,+\infty)$ and $A \in \tau_{a_{m}}$ such that $A=E\left(\bmod \mathcal{L}^{n}\right)$.

Proof. Let $q_{1}, q_{2}, \ldots \in \mathbb{Q}^{n} \cap(0,1)^{n}$ and $r_{1}, r_{2}, \ldots \in(0,1)$ be sequences such that

$$
n \omega_{n} r_{k}^{n-1} \leq 2^{-k}, \quad \overline{B\left(q_{k}, r_{k}\right)} \subset E_{k}^{\circ}
$$

for all $k=1,2, \ldots$, where $E_{1}:=[0,1]^{n}$ and (for $k \geq 2$ )

$$
E_{k}:=[0,1]^{n} \backslash\left(B\left(q_{1}, r_{1}\right) \cup \cdots \cup B\left(q_{k-1}, r_{k-1}\right)\right) .
$$


Define

$$
E:=\bigcap_{k=0}^{+\infty} E_{k}=[0,1]^{n} \backslash \bigcup_{k=0}^{+\infty} B\left(q_{k}, r_{k}\right)
$$

Then $E$ is closed and, by appropriately choosing the sequence $\left\{q_{k}\right\}$, we can always assume that $E^{\circ}=\emptyset$. Moreover one has

$$
\mathcal{L}^{n}(E)=1-\sum_{k=0}^{+\infty} \omega_{n} r_{k}^{n} \geq 1-\frac{1}{n} \sum_{k=0}^{+\infty} n \omega_{n} r_{k}^{n-1} \geq 1-\frac{1}{n} \sum_{k=0}^{+\infty} 2^{-k}=1-\frac{1}{n}>0 .
$$

Since $E_{k} \rightarrow E$ in measure, from [3, Proposition 3.38]) it follows that $E$ has finite perimeter:

$$
P(E) \leq \liminf _{k \rightarrow \infty} P\left(E_{k}\right)=2 n+\sum_{k=1}^{+\infty} n \omega_{n} r_{k}^{n-1} \leq 2 n+\sum_{k=1}^{+\infty} 2^{-k}=2 n+1 .
$$

Finally, we will prove (3) with a reductio ad absurdum. For this purpose, we assume that there exist $m \geq n$ and $A \in \tau_{a_{m}}$ such that

$$
A=E \quad\left(\bmod \mathcal{L}^{n}\right) .
$$

Then:

- One has $A^{\circ}=\emptyset$. If not $A$ should include a nontrivial open ball $B$, hence

$$
0=\mathcal{L}^{n}(B \backslash A)=\mathcal{L}^{n}(B \backslash E) .
$$

Since $B \backslash E$ is open, this equality shows that $B \backslash E=\emptyset$ i.e. $B \subset E$, which contradicts $E^{\circ}=\emptyset$;

- From Proposition 3.5 and the previous point it follows that $\mathcal{L}^{n}(A)=0$. Hence $\mathcal{L}^{n}(E)=0$ and this provides the absurd.

Remark 4.1. For $m>m_{0}$, Proposition 4.3 holds with $E:=F_{m}$. Indeed (1) and (2) follows at once from Proposition 4.1, while the proof of (3) remains the same.

\section{REFERENCES}

[1] G. Alberti: A Lusin Type Theorem for Gradients. J. Funct. Anal. 100, 110-118 (1991).

[2] L. Ambrosio, V. Caselles, S. Masnou, J.-M. Morel: Connected components of sets of finite perimeter and applications to image processing. J. Eur. Math. Soc. 3, 39-92 (2001).

[3] L. Ambrosio, N. Fusco, D. Pallara: Functions of bounded variation and free discontinuity problems. Oxford Mathematical Monographs, Oxford University Press Inc. 2000.

[4] D.G. Caraballo: B2-convexity implies strong and weak lower semicontinuity of partitions of $\mathbb{R}^{n}$. Pacific J. Math. 253 (2011), no. 2, 321-348.

[5] S. Delladio: Functions of class $C^{1}$ subject to a Legendre condition in an enhanced density set. Rev. Matem. Iberoam. 28 (2012), n. 1, 127-140.

[6] S. Delladio: A short note on enhanced density sets. Glasgow Math. J. 53 (2011), n. 3, 631-635.

[7] S. Delladio: A Whitney-type result about rectifiability of graphs. Riv. Mat. Univ. Parma, Vol. 5 (2014), 387-397. 
[8] S. Delladio: Some new results about the geometry of sets of finite perimeter. To appear on Proc. Royal Soc. Edinburgh.

[9] G. Dolzmann, S. Müller: Microstructures with finite surface energy: the two-well problem. Arch. Rational Mech. Anal. 132 (1995), 101-141.

[10] A. Figalli, F. Maggi: On the shape of liquid drops and crystals in the small mass regime. Arch. Ration. Mech. Anal. 201 (2011), n. 1, 143-207.

[11] A. Figalli, F. Maggi, A. Pratelli: A mass transportation approach to quantitative isoperimetric inequalities. Invent. Math. 182 (2010), n. 1, 167-211.

[12] J.H.G. Fu: Erratum to "Some Remarks On Legendrian Rectifiable Currents". Manuscripta Math. 113, n. 3, 397-401 (2004).

[13] Y. Gousseau, J.-M. Morel: Are natural images of bounded variation? SIAM J. Math. Anal. 33 (2001), n. 3, 634-648

[14] B. Kirchheim: Lipschitz minimizers of the 3-well problem having gradients of bounded variation. Preprint 12, Max Planck Institute for Mathematics in the Sciences, Leipzig, 1998.

[15] J. Lukes, J. Maly and L. Zajicek: Fine topology methods in real analysis and potential theory. Lect. Notes in Math. 1189, Springer Verlag 1986.

[16] D. Pavlica, L. Zajcek: Morse-Sard theorem for d.c. functions and mappings on $\mathbb{R}^{2}$. Indiana Univ. Math. J. 55 (2006), n. 3, 1195-1207. 